\title{
Protecting generalism: moving on from evidence-based medicine?
}

\author{
Joanne Reeve
}

\author{
ABSTRACT \\ Quality of decision making in modern health care is \\ defined with reference to evidence-based medicine. \\ There are concerns that this approach is insufficient \\ for, and may thus threaten the future of, generalist \\ primary care. We urgently need to extend our account \\ of quality of knowledge use and decision making in \\ order to protect and develop the discipline. Interpretive \\ medicine describes an alternative framework for use in \\ generalist care. Priorities for clinical practice and \\ research are identified. \\ Keywords \\ evidence-based medicine; generalism; quality.
}

Evidence-based medicine (EBM) was first described as an approach to teaching the practice of medicine. ${ }^{1}$ Twenty-five years on, it has become an assumed standard of 'best'" or even 'reasonable' ${ }^{3}$ practice. EBM recognised a need to support healthcare professionals in maintaining an up-to-date working account of the ever-expanding scientific knowledge about illness and health care. Defined as the 'judicious application of best evidence in making clinical decisions about this individual', ${ }^{4}$ EBM acknowledges both the value and necessity of external research evidence integrated with clinical expertise in clinical decision making.,5 Good doctors use both; neither alone is enough. ${ }^{4} \mathrm{EBM}$ describes an 'ideal of practice' ${ }^{6}$ and few GPs would reject a principle of evidence-informed decision making. ${ }^{3,7}$

It is in the detail and implementation of that ideal that problems have arisen. Sackett defined best

J Reeve, BClinSci, PhD, MPH, MRCGP, DipFPH, NIHR clinician scientist in Primary Care, Division of Primary Care, University of Liverpool, Liverpool.

Address for correspondence

Dr Joanne Reeve, Division of Primary Care, University of Liverpool, Waterhouse Building, Block B, 1st Floor, B122, 1-5 Brownlow Street, Liverpool, L69 3GL.

E-mail: joanne.reeve@liv.ac.uk

Submitted: 6 October 2009; Editor's response:

3 January 2010; final acceptance: 4 February 2010.

OBritish Journal of General Practice 2010; 60: 521-523.

DOI: 10.3399/bjgp10X514792 evidence as that which was 'clinically relevant', ${ }^{4}$ but with heavy emphasis placed on the approaches of clinical epidemiology, ${ }^{4,5}$ and randomised trials of interventions. ${ }^{4}$ The evidence-based practice movement has embraced the philosophy of positivist science. $^{6}$ 'Hierarchies' of evidence privilege knowledge from what some consider to be a narrow methodological perspective; ${ }^{3,6}$ an approach which is contested. ${ }^{6}$ As health policy identified evidence-based practice as a tool to address variation and quality in care, the ideals of EBM have developed into what Harrison describes as 'scientific bureaucratic medicine'. ${ }^{8}$ With concerns that healthcare professionals are 'too busy' to practice EBM, external knowledge experts following defined rules for interpretation created definitive guidelines for clinical decision making. ${ }^{8}$ The disadvantages of constrained protocol-driven care are recognised by the profession; ${ }^{7}$ but still form a new formative normative framework for clinical practice. ${ }^{8,9}$ Problems with implementation shouldn't lead us to reject the concept of EBM, but do suggest the need for critical review and development.

EBM was developed within the disease-oriented setting of specialist secondary care. Yet generalist practice is more than disease-focused care delivered in a community setting. It is a different approach to understanding and addressing health and illness. Generalism describes a philosophy of practice which is person, not disease, centred; continuous, not episodic; integrates biotechnical and biographical perspectives; and views health as a resource for living and not an end in itself. ${ }^{10-13}$ Stange has called for a revival of the generalist approach to address growing concerns about the inefficiency, ineffectiveness, and inequity of fragmented health care. ${ }^{14} \mathrm{He}$ highlights the need to understand specific events in their broader context, integrating biomedical evidence with a reflexive and interpretative approach that acknowledges the complexity of individual human experience. ${ }^{11,12,14}$ With evidence that the current model of EBM as a normative model of practice may be contributing to a shift away from generalist principles to a more disease-focused model of care, ${ }^{10,14,15}$ we need an alternative approach to defining quality of knowledge use within generalist practice.

I have therefore described interpretive medicine as an alternative account of knowledge use in generalist 


\section{How this fits in}

The principles of evidence-based medicine define quality decision making in specialist care. Generalist care lacks an equivalent framework adequate to define quality of person-centred decision making. This gap threatens the future of the discipline. Interpretive medicine describes an alternative framework for use in generalist care. approach to the application of knowledge in supporting health care. Practising clinicians, and indeed many patients and carers, will recognise these issues as part of the daily complex decision making of generalist practice. However, this complex and skilled task is undersupported and undervalued within increasingly specialist and fragmented models of illness and care.

Interpretive medicine represents an important shift in emphasis from the application of evidence in decision making, to the generation of individualised knowledge as an essential quality marker of generalist care. An extensive body of professional writing addresses the skills and processes needed for individualised care: in applying clinical judgement to address the limitations of knowledge; in dealing with the epistemological uncertainty that comes in seeking to apply 'certain' knowledge derived from the study of disease in populations to understand an individual patient; ${ }^{20}$ and in integrating biological and biographical accounts of illness. ${ }^{12}$ The field of narrative medicine provides one such account of how to undertake person-centred practice..$^{21,22}$ Even with this rich heritage, we must not be complacent about the ongoing need for education and training to support the development and maintenance of skills of interpretive medicine. ${ }^{23}$ However, my concern within this paper is to highlight the need to now focus on evaluation of the quality and outcomes of generalist care. We need a framework to describe quality of knowledge generation and use in interpretive care, and hence the implications for patient outcomes.

Interpretive medicine describes a process of knowledge generation: knowledge which may be subjected to similar tests of trustworthiness as that generated by research. Drawing on Doucet and Mauthner's ${ }^{24}$ assertion that knowledge should be judged by reviewing the processes and assumptions by which it is produced, rather than solely by its proximity to truth, I propose two urgent priorities for clinicians and academics in developing interpretive medicine as a framework to support generalist practice.

First, we need a tool to support front-line practitioners in maintaining skills and confidence in the interpretive process. I propose the development of a knowledge appraisal tool to support evaluation of the processes and outcomes of knowledge generation within the context of a person-centred consultation. ${ }^{10}$ The tool should support practitioners in considering what data is collected (a patient narrative, practitioner narrative, and clinical signs). It should promote reflection on the interpretive stance of both patient and doctor (understanding illness from a biomedical and/or biographical perspective). Reflection should lead to a critical analysis of alternative interpretations, 
opportunities missed, and the potential impact on outcomes. An interesting development could include reflection with the patient, possibly with an appraiser as facilitator. Criteria for quality, similar to those described for qualitative research,,$^{25}$ provide a tool to support external review of the interpretive process ${ }^{10}$ and thus address concerns about unsupported, and undervalued, interpretive practice.

Second, we need research into the patient outcomes resulting from different approaches to knowledge use, and particularly an interpretive approach in the care of patients with complex needs. Through consultation analysis, we need to be able to identify interpretive (generalist) and non-generalist approaches to care so that we can evaluate the impact of each approach on individual patient outcomes. We need to understand when an interpretive approach is of value, and when more disease-focused (protocol-informed) care is useful for this individual. Key questions include who needs generalist (beyond protocol) care, when, and with what outcomes? Answers to these are fundamental to planning the future delivery of care.

The legitimate use of knowledge is a defining aspect of modern clinical practice, ${ }^{10}$ and shapes ideas about quality in practice. The ability to integrate knowledge to provide individualised care should be seen as a marker of quality in generalist practice. ${ }^{10}$ The profession should be judged not purely by what knowledge it uses, but by the way it uses it. We therefore need to shift the gaze from easier to measure but limited accounts of practice based on the application of certain knowledge to a more appropriate assessment of knowledge use, in order to strengthen and preserve core elements of the discipline and promote and support the health needs of the public.

\section{Funding}

At the time of writing, Joanne was employed as an NIHR Clinical Lecturer in Primary Care at the University of Manchester.

\section{Competing interests}

The authors have stated that there are none.

\section{Acknowledgements}

The authors wishes to give thanks to Chris Dowrick and to the journal peer reviewers for helpful comments on an earlier draft.

\section{Discuss this article}

Contribute and read comments about this article on the Discussion Forum: http://www.rcgp.org.uk/bjgp-discuss

\section{REFERENCES}

1. Evidence-Based Medicine Working Group. Evidence-based medicine: a new approach to teaching the practice of medicine. JAMA 1992 268(17): 2420-2425.

2. Lambert $\mathrm{H}$, Gordon EJ, Bogdan-Lowis EA. Introduction: gift horse or Trojan horse? Social science perspectives on evidence-based health care. Soc Sci Med 2006; 62(11): 2613-2620.

3. Goldenberg M. On evidence and evidence-based medicine: lessons from the philosophy of science. Soc Sci Med 2006; 62(11): 2621-2632.
4. Sackett DL, Rosenberg WM, Muir Grey JA, et al. Evidence-based medicine: what it is and isn't. BMJ 1996; 312(7023): 71-72.

5. Straus SE, Richardson WS, Glasziou P, Haynes RB. Evidence-based medicine. How to practice and teach EBM. (3rd edn). Edinburgh: Elsevier Churchill Livingstone, 2005.

6. Marks DF. Perspectives on evidence-based practice. London: Health Development Agency, 2002.

http://www.nice.org.uk/aboutnice/whoweare/aboutthehda/evidencebas e/publichealthevidencesteeringgroupproceedings/perspectives_on_evid ence_based_practice.jsp (accessed 27 May 2010)

7. Mant D. The problem with usual care. Br J Gen Pract 2008; 58(556): 755-756.

8. Harrison S, Moran M, Wood B. Policy emergence and policy convergence: the case of 'scientific-bureaucratic medicine' in the United States and United Kingdom. Br J Polit Int Relat 2002; 4(1): 1-24.

9. Harrison S. New Labour, Modernisation and the Medical Labour Process. J Soc Pol 2002; 31(3): 465-485.

10. Reeve J. Interpretive medicine: supporting generalism in a changing primary care world. London: Royal College of General Practitioners Occasional Paper Series, 2010.

http://www.rcgp.org.uk/bookshop/info_1_9780850843286.html (accessed 11 June 2010).

11. Stange KC. The generalist approach. Ann Fam Med 2009; 7(3): 198-203.

12. Gunn JM, Palmer VJ, Naccarella L, et al. The promise and pitfalls of generalism in achieving the Alma-Ata vision of health for all. Med J Aust 2008; 189(2): 110-112.

13. Declaration of Alma-Ata. International conference on primary health care Alma-Ata, USSR, 6-12 September 1978. http://www.who.int/publications/almaata_declaration_en.pdf (last accessed 01/02/2010)

14. Stange KC. The problem of fragmentation and the need for integrative solutions. Ann Fam Med 2009; 7(2): 100-103.

15. Checkland K, Harrison S, McDonald R, Grant S. Biomedicine, holism and general medical practice: responses to the 2004 general practitioner contract. Sociol Health Illn 2008; 30(5): 788-803.

16. Willis J. The paradox of progress. Oxford: Radcliffe Medical Press, 1995.

17. Dowrick C. Beyond depression: a new approach to understanding and management. (2nd edn). Oxford: OUP, 2009.

18. Griffiths F, Anto N, Chow E, Manazar U. Understanding the diversity and dynamics of living with diabetes: a feasibility study focusing on the case. Chronic Illn 2007; 3(1): 29-45.

19. Reeve J, Lloyd-Williams M, Payne S, Dowrick CF. Towards a reconceptualisation of the management of distress in palliative care patients: the Self-Integrity Model. Prog Palliat Care 2009; 17(2): 51-60.

20. Fox RC. Medical uncertainty revisited. In: Bendelow G, Carpenter M, Vantier C, Williams S (eds). Gender health and healing. The public/private divide. London: Routledge, 2002: 236-253.

21. Launer J. Narrative-based primary care. A practical guide. Oxon: Radcliffe Medical Press Ltd, 2002.

22. Greenhalgh T, Hurwitz B. Narrative based medicine. Dialogue and discourse in clinical practice. London: BMJ Books, 1998.

23. Dowrick C, May C, Richardson M, Bundred P. The biopsychosocial model of general practice: rhetoric or reality? Br J Gen Pract 1996; 46(403): 105-107.

24. Doucet A, Mauthner N. Knowing responsibly: linking ethics, research practice and epistemology. In: Mauthner M, Birch M, Jessop J, ller T (eds). Ethics in qualitative research. London: Sage Publications Ltd, 2002.

25. Maxwell J. Understanding and validity in qualitative research. In: Huberman A, Miles M (eds). The qualitative researcher's companion. Thousand Oaks, California: Sage Publications Ltd, 2002.

\section{By the same author}

Occasional Paper 88 - Interpretive medicine: Supporting generalism in a changing primary care world. Dr Joanne Reeve, RCGP: 2010

This Occasional Paper looks at current models of knowledge use in general practice. It contains a critical review of the literature, and draws on the author's empirical research and reflections from clinical practice. It is available for purchase via the RCGP Online Bookstore: www.rcgp.org.uk/bookshop, priced $£ 15.00$ (RCGP members: £13.50). 